\title{
A!
}

This is an electronic reprint of the original article.

This reprint may differ from the original in pagination and typographic detail.

Haneda, Katsuyuki; Heino, Mikko; Jarvelainen, Jan

\section{Total Array Gains of Polarized Millimeter-Wave Mobile Phone Antennas}

Published in:

2018 European Conference on Networks and Communications, EuCNC 2018

DOI:

10.1109/EuCNC.2018.8442687

Published: 20/08/2018

Document Version

Peer reviewed version

Please cite the original version:

Haneda, K., Heino, M., \& Jarvelainen, J. (2018). Total Array Gains of Polarized Millimeter-Wave Mobile Phone Antennas. In 2018 European Conference on Networks and Communications, EuCNC 2018 (pp. 167-171). [8442687] (European Conference on Networks and Communications). IEEE.

https://doi.org/10.1109/EuCNC.2018.8442687

This material is protected by copyright and other intellectual property rights, and duplication or sale of all or part of any of the repository collections is not permitted, except that material may be duplicated by you for your research use or educational purposes in electronic or print form. You must obtain permission for any other use. Electronic or print copies may not be offered, whether for sale or otherwise to anyone who is not an authorised user. 
This is the accepted version of the original article published by IEEE.

(C) 2018 IEEE. Personal use of this material is permitted. Permission from IEEE must be obtained for all other uses, in any current or future media, including reprinting/republishing this material for advertising or promotional purposes, creating new collective works, for resale or redistribution to servers or lists, or reuse of any copyrighted component of this work in other works. 


\title{
Total Array Gains of Polarized Millimeter-Wave Mobile Phone Antennas
}

\author{
Katsuyuki Haneda, Mikko Heino and Jan Järveläinen \\ Department of Electronics and Nanoengineering, \\ Aalto University School of Electrical Engineering, Espoo, Finland \\ E-mail: see http://ele.aalto.fi/en/contact/
}

\begin{abstract}
This paper studies a gain of an antenna array implemented on a mobile device operating at a millimeter-wave radio frequency. Assuming that mobile phones at millimeterwave range operate with a single transceiver chain and analog beamforming like phased arrays, its total array gain is attributed to average gains of antenna elements and signal precoding or combining gains in excess to received power from a single-element dual-polarized omni-directional antenna. The total array gain circumvents the ambiguity of conventional array gain which cannot be uniquely defined as there are multiple choices of a reference single-element antenna in an array. Different polarized 8 -element patch antenna arrays implemented on a mobile phone chassis, i.e., uniform linear array (ULA) and distributed array (DA) operating at $60 \mathbf{G H z}$, are studied. The antenna elements are placed so that they cover vertical, slanted or horizontal polarizations. The gain is evaluated for different orientations of the chassis along with effects of a body torso and a finger of a person operating the phone. The gain in a small-cell scenario shows that DA achieves higher gains than ULA regardless of polarization states of antenna elements at the base and mobile stations, and of the existence of line-of-sight in the links. Antenna polarizations do not make much impact on the total array gain as random orientation of a mobile phone and finger shadowing modifies the polarization states. The results show that antenna array geometry is more influential design aspect than polarization when a single transceiver chain is considered.
\end{abstract}

Index Terms-Millimeter-wave, antenna array, mobile phone, array gain, user effect, body shadowing.

\section{INTRODUCTION}

New generations of cellular mobile networks, called the fifth-generation radios, have been studied intensively both in the industry and academy. The new generation of networks exploits radio frequencies higher than $6 \mathrm{GHz}$ actively for higher peak data rates and network throughput. One of the practical issues in cellular mobile radios operating at higher frequency bands is its coverage. Higher radio frequencies typically have smaller service coverage compared to lower frequency radio, particularly in non and obstructed line-ofsight conditions in light of greater signal losses in diffraction and penetration. Mathematical models and tools to predict losses due to radio propagation have been studied in the previous years extensively, e.g., [1]. In contrast, possible gains or losses attributed to antennas implemented on mobile phone devices have received less attention, particularly under the presence of fingers and human bodies of mobile users [2]-[6]. This paper therefore sheds lights on gains of practical mobile phone antennas operating at higher frequencies than $6 \mathrm{GHz}$, particularly at millimeter-waves (mm-waves). In concrete, we reveal the achievable gain of $60 \mathrm{GHz}$ polarimetric antenna arrays at a mobile phone that work as a phased antenna with a single transceiver chain. We study a gain of polarimetric array antennas under realistic operational conditions with influence of finger, body and multipath channels. A total array gain defined in [6] is used for the study. The gain defines a received power at a mobile array that it can extract from multipath radio channels in excess to a single-element dual-polarized omni-directional antenna. The definition is analogous to the mean effective gain for a single-element antenna [7], [8], but the present paper studies gains of an array. Pathloss of a radio channel is called omni-directional pathloss when a base station (BS) and mobile station (MS) is equipped with omnidirectional antennas. The omni-directional pathloss has been discussed popularly in mm-wave channel modeling, including the recently established 3GPP standard channel model for new radios [1]. In contrast to the conventional array gain which cannot be defined uniquely depending on the choice of a reference single-element antenna in an array, the total array gain is uniquely defined and allows us to compare arrays formed by different antenna elements and hence polarizations. We evaluate the total gain of $60 \mathrm{GHz}$ antenna arrays at MS: an 8element uniform linear array (ULA) and distributed array (DA) both consisting of patch antennas with different polarization combinations. The evaluation is based on electromagnetic field simulations for antennas and measurement-based ray-tracing propagation simulations in a small-cell scenario at an airport. Finger and human torso effects on radiation characteristics of the considered MS antennas are taken into account. This paper is a companion volume to [6]; the cited paper studies only MS antenna arrays with slanted polarizations, while the present paper adds insights about differently polarized antenna arrays at BS and MS. Furthermore, this paper shows that the total array gain yields a channel capacity straightforwardly by combining it with the omni-pathloss and a receiver noise level.

The rest of the paper is organized as follows: Section II introduces different polarized antenna arrays on MS and their varying postures. Section III describes the site-specific radio propagation simulation supported by experiments. Section IV first derive the total array gain and compare it for the considered antenna arrays. Section V summarizes the main 


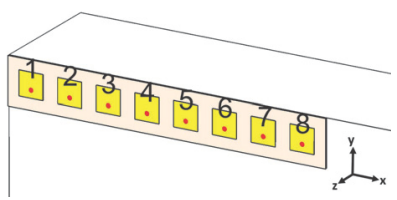

(a)

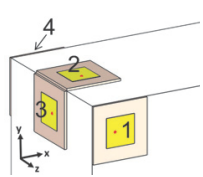

(c)

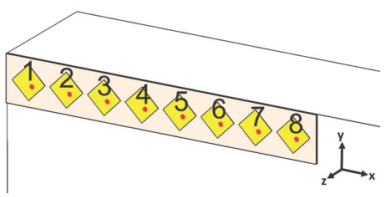

(b)

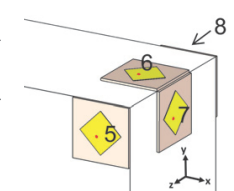

(f)
Fig. 1. Arrangement of patch antennas on a mobile phone chassis with dimensions of $150 \times 75 \times 8 \mathrm{~mm}^{3}$ in length, width and thickness: uniform linear arrays with (a) vertical and (b) slanted polarizations; distributed arrays with (c) and (d) vertical or horizontal and (e) and (f) slanted polarizations.

conclusions obtained in this paper.

\section{II. $60 \mathrm{GHz}$ Mobile Phone Antennas}

\section{A. Antenna Arrays}

We consider different antenna arrays as practical examples of $60 \mathrm{GHz}$ arrays for MS, i.e., ULA and DA with vertical, horizontal or slanted polarizations to the ground when a phone chassis is at a standing position on the ground as illustrated in Fig. 1. The arrays are arranged by 8 square patch antennas. The neighboring antennas of ULA radiate the same vertical or slanted polarizations, or both vertical and horizontal polarizations in an alternating manner. The antenna elements are separated by half the free space wavelength ${ }^{1}$. The array is installed at the left-top corner of an MS chassis. In DA, patch antennas are installed at each side of the two top corners of the chassis. The chassis has dimensions of $150 \times 75 \times 8 \mathrm{~mm}^{3}$ in length, width and thickness and is considered to be a ground plane of the antennas. The patch antennas are implemented on a $0.127 \mathrm{~mm}$ thick Rogers 5880 substrate with a relative permittivity of $\epsilon_{\mathrm{r}}=2.2$ and a $17 \mu \mathrm{m}$ thick copper layer. The antennas have a maximum broadside gain of $G_{\mathrm{b}}=8 \mathrm{~dB}$. The whole structure is simulated in CST Microwave Studio. The far-field radiation patterns of the antenna element show that the maximum backlobe radiation is weaker than the main lobe gain by at least $20 \mathrm{~dB}$ due to the chassis serving as an electrically large ground plane. DA has more uniform illumination of the entire solid angle since the broadsides of antenna elements point different directions. It is however harder for the DA to leverage the maximum array gain because antennas' broadsides point different directions.

\section{B. Orientations of the Mobile Phone}

Different orientations of MS chassis are taken into account to analyze realistic operational scenarios. Figure 2 shows the coordinate system and a base orientation of MS where the long-side of a phone chassis is along the $y$-axis, while the display faces $+z$-direction. Orientation of MS is determined

\footnotetext{
${ }^{1}$ The spacing is greater than half the wavelength of the antenna substrate.
}

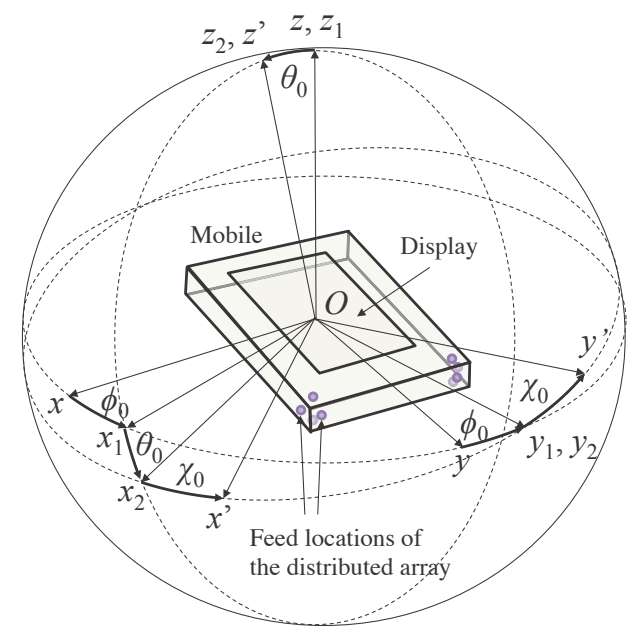

Fig. 2. Coordinate system definition of a mobile phone and its orientation The angles $\left(\phi_{0}, \theta_{0}, \chi_{0}\right)$ define the rotation of a mobile phone.

by rotating the coordinate system through three angles, $\phi_{0}$, $\theta_{0}$ and $\chi_{0}$ in Fig. 2, while fixing MS chassis. The three angles rotates the original coordinate system $(x, y, z)$ around $z, y_{1}$ and $z_{2}$ axes, respectively, so that the rotated coordinate system becomes $\left(x^{\prime}, y^{\prime}, z^{\prime}\right)$ [9], Appendix A2. The three angles $\left(\phi_{0}, \theta_{0}, \chi_{0}\right)$ are set such that the longitudinal axis of MS chassis is along a line of every $45^{\circ}$ azimuth between $0^{\circ}$ and $315^{\circ}$ and of the polar angle at $0^{\circ}, 45^{\circ}$ and $90^{\circ}$. The radiation patterns of antenna elements on the rotated coordinate system, $\boldsymbol{E}_{\mathrm{m}}$, are derived from those on the original coordinate system as detailed in Appendix of [6].

\section{Finger Effects}

We also consider cases when a finger covers one of the antenna elements. A finger covering an antenna gives rise to the reduction of broadside gains of patch antennas by 18 to $25 \mathrm{~dB}$ [10]. A finger is modeled as a single layer with an elliptic cross section and is separated by $3 \mathrm{~mm}$ from the antenna in our simulations to avoid severe reduction of radiation efficiency. When a fingertip points to one of the antennas, other antennas also suffer from shadowing due to the finger, but not as severely as the one covered with the finger. The worst input impedance matching at $60 \mathrm{GHz}$ among patch antennas is $9.2 \mathrm{~dB}$ of return loss under the presence of a finger for ULA, while the same is $14.3 \mathrm{~dB}$ for DA. The return losses are much greater without a finger. The worst isolation between patch antennas is 16.0 and $35.3 \mathrm{~dB}$ for ULA and DA, respectively, under the presence of a finger. The isolation is higher if there is no finger.

\section{Radio Propagation Simulations}

We introduce a ray-tracer that is qualified to produce realistic multipath channels. The simulated multipath channels are compared with measurements to justify their validity.

\section{A. Optimization of Ray-Tracer}

An in-house ray-tracer [11] is applied to a check-in hall of an airport as a representative small-cell scenario, whose 


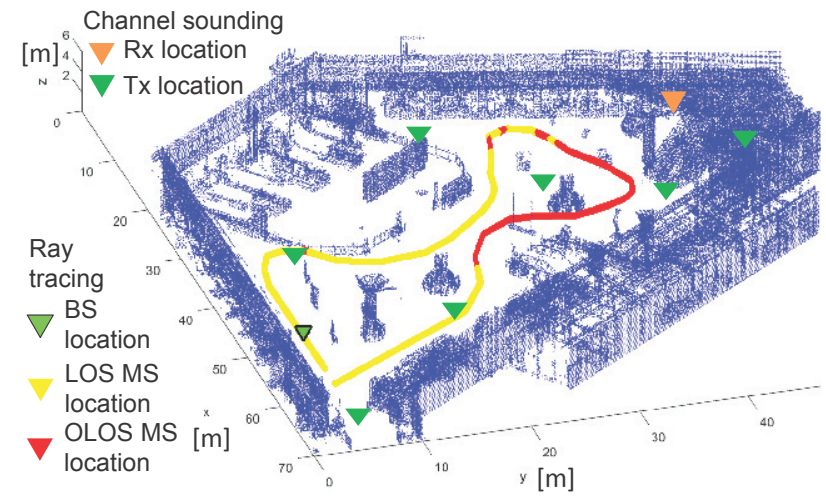

Fig. 3. Floor plan of the small-cell site in an airport.

floor plan is depicted in Fig. 3. The ray-tracer is based on accurate descriptions of the environment in the form of point clouds, obtained by laser scanning. It is capable of simulating relevant propagation mechanisms such as specular reflections, diffraction, diffuse scattering and shadowing. Specular reflections are first identified by finding points lying inside the Fresnel zone between MS and images of BS, and then checking if a normal vector of a local surface formed by a group of points supports the specular reflection. Once identified, the reflection coefficients are calculated using the Fresnel equations. Shadowing objects are similarly detected by searching for points within the Fresnel zone for a given path. The ray-tracer provides azimuth and polar angles of arriving multipath components (MPCs) at MS as well as the co-polarized magnitude of path gain and propagation delay time, i.e., $\left\{\phi_{l}, \theta_{l}, \alpha_{l}^{V V}, \tau_{l}\right\}_{l=1}^{N_{\mathrm{p}}}$, as outputs where $N_{\mathrm{p}}$ is a number of MPCs in a link. The magnitude is given by

$$
\alpha^{V V}=\frac{\Gamma}{\sqrt{L_{\mathrm{a}} L_{\mathrm{body}}}} \cdot \frac{\exp \left(-\mathrm{j} 2 \pi \tau f_{\mathrm{c}}\right)}{4 \pi \tau f_{\mathrm{c}}},
$$

where $\Gamma$ is a product of all complex reflection coefficients along a propagation path, $f_{\mathrm{c}}$ is a carrier frequency of the simulation, $L_{\mathrm{a}}$ and $L_{\text {body }}$ are extra losses due to small objects and a human torso, respectively, which are defined later. In the present simulations, we take into account the LOS path as well as first and second order specular reflections. Diffuse scattering is found to be of minor effects in the present case.

The ray-tracer is optimized for the small-cell scenario by comparing with measured channels at the same site [12]. Spatio-temporal characteristics of radio channels at $60 \mathrm{GHz}$ are measured for 12 links between a fixed receive (Rx) antenna and a varying transmit $(\mathrm{Tx})$ antenna locations. We set permittivity $\varepsilon_{\mathrm{r}}$ of materials so that the reproduced channels resemble the measured ones. Optimum $\varepsilon_{\mathrm{r}}$ is found by first calculating the path amplitude with different $\varepsilon_{\mathrm{r}}$ ranging from 2 to 6 , then deriving a band-limited power delay profile (PDP) from the paths and finally minimizing the difference between measured and simulated delay spreads. The shadowing loss due to small objects in the environment is chosen heuristically, $L_{\mathrm{a}}=20 \mathrm{~dB}$. Paths propagating through walls are assigned with very high attenuation losses because these paths do

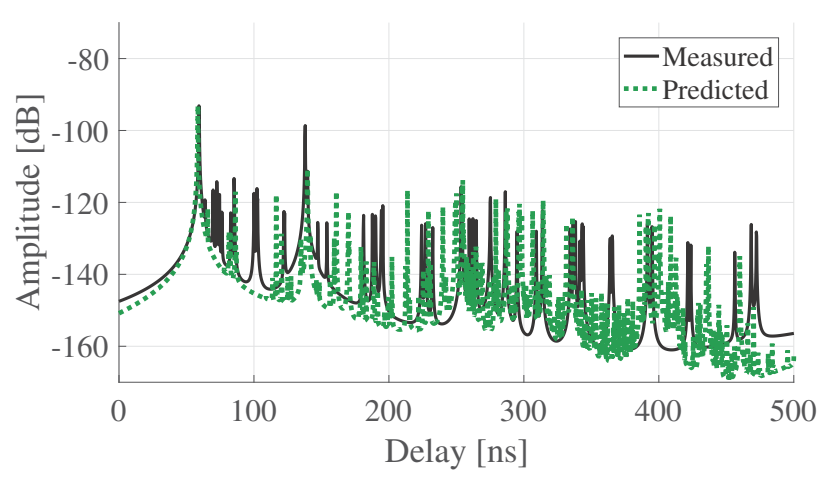

(a)
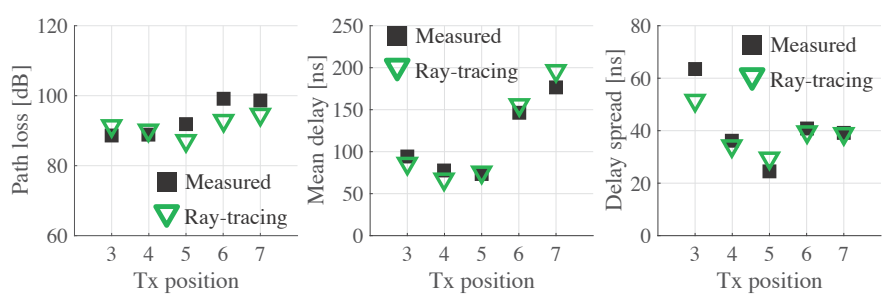

(b)

Fig. 4. (a) Measured and simulated PDP for Tx4. (b) Large-scale parameters for measured and optimized ray-traced channels at $60 \mathrm{GHz}$.

not contribute to the received power. Optimization yielded $\varepsilon_{\mathrm{r}}=3.6$, leading to agreement of the measured and simulated PDP shown in Fig. 4(a) for one of the links. A comparison between measured and simulated pathloss, mean delay and delay spreads is shown in Fig. 4(b).

\section{B. Running the Ray-Tracer}

With the optimum parameters of ray-tracing, MPCs are generated for BS and MS locations defined in Fig. 3. The BS is placed $1 \mathrm{~m}$ from a wall at a height of $5.7 \mathrm{~m}$. The MS is placed at a height of $1.5 \mathrm{~m}$ at every $0.6 \mathrm{~m}$ over a route. In total, 2639 links are simulated, including 1816 LOS and 823 obstructed LOS (OLOS). The polarimetric complex amplitudes of each MPC are generated statistically from $\alpha^{V V}$ estimates of the ray-tracer as $\alpha^{H H}=\alpha^{V V}$ and

$$
\alpha^{H V}=\alpha^{V H}=\alpha^{V V} / \mathrm{XPR},
$$

where $\alpha^{V H}$ for example denotes a complex amplitude of a horizontally-transmitted and vertically-received path; XPR is a cross-polarization ratio (XPR) of an MPC modeled from polarimetric channel sounding [13] as

$$
\begin{gathered}
\left.\mathrm{XPR}\right|_{\mathrm{dB}} \sim \mathcal{N}\left(\mu_{2}\left(\left.L_{\mathrm{ex}}\right|_{\mathrm{dB}}\right), \sigma_{2}^{2}\right), \\
\mu_{2}\left(\left.L_{\mathrm{ex}}\right|_{\mathrm{dB}}\right)= \begin{cases}\left.\alpha_{2} \cdot L_{\mathrm{ex}}\right|_{\mathrm{dB}}+\beta_{2}, & \text { if }\left.L_{\mathrm{ex}}\right|_{\mathrm{dB}} \leq-\beta_{2} / \alpha_{2} \\
0, & \text { if }\left.L_{\mathrm{ex}}\right|_{\mathrm{dB}}>-\beta_{2} / \alpha_{2}\end{cases}
\end{gathered}
$$

where $\mu_{2}\left(\left.L_{\mathrm{ex}}\right|_{\mathrm{dB}}\right)$ is the mean, $\sigma_{2}^{2}$ is the variance of the XPR model; $\alpha_{2}=-0.6, \beta_{2}=35$ and $\sigma_{2}=4$ are used [13]. The excess loss $\left.L_{\mathrm{ex}}\right|_{\mathrm{dB}}$ of the MPC is defined as

$$
\left.L_{\mathrm{ex}}\right|_{\mathrm{dB}}=-20 \log _{10}\left(\left|\alpha^{V V} \cdot 4 \pi \tau f_{\mathrm{c}}\right|\right) .
$$




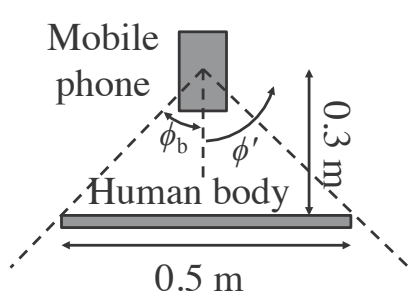

(a)

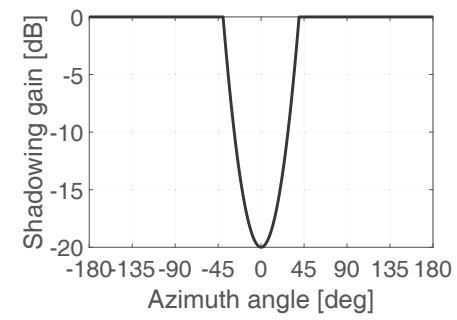

(b)
Fig. 5. (a) Geometry of the mobile phone and human torso. (b) Canonical model of shadowing losses at $60 \mathrm{GHz}$ for a human torso.

\section{Human Torso Shadowing}

At $60 \mathrm{GHz}$, it is necessary to include a link blockage effect due to a human body holding an MS. A simple canonical model of the link blockage due to a human body is used. A relative geometry of the human body to an MS chassis is defined in Fig. 5(a), where the width of the human body and the separation between the body and the mobile is 0.5 and $0.3 \mathrm{~m}$, respectively. The human blockage loss is defined by

$$
\left.L_{\text {body }}\right|_{\mathrm{dB}}=\max \left(0,\left.L_{\mathrm{b}}\right|_{\mathrm{dB}}\left\{1-\left(\frac{\phi-\left(\phi_{0}-\pi\right)}{\phi_{\mathrm{b}}}\right)^{2}\right\}\right) \text {, }
$$

where $\phi$ is the azimuth angle of arrival of an MPC, $\phi_{\mathrm{b}}=39.8^{\circ}$ is the azimuth angle of the body torso seen from the mobile as defined in Fig. $5(\mathrm{a}) ; L_{\mathrm{b}}=20 \mathrm{~dB}$ is the maximum body shadowing loss. The model provides different losses depending on the azimuth angle of arrival of MPCs.

\section{TOTAL ARRAY GAIN}

\section{A. Definition}

It is possible to define array gain of the antenna at MS locations after we have the polarimetric complex gain of the antenna radiation patterns, $\boldsymbol{E}_{\mathrm{m}}$, and parameters of MPCs, $\left\{\phi_{l}, \theta_{l}, \boldsymbol{\alpha}_{l}, \tau_{l}\right\}_{l=1}^{N_{\mathrm{p}}}$, where $\boldsymbol{\alpha}_{l} \in \mathbb{C}^{2 \times 2}$ is a polarimetric complex gain of an $l$-th MPC. Assuming downlink, the output voltage $\boldsymbol{y}$ observed at an MS antenna array is expressed as

$$
\boldsymbol{y}=\boldsymbol{h} x+\boldsymbol{n},
$$

where $x$ is an input voltage to a single-antenna base station, $\boldsymbol{n}, \boldsymbol{h} \in \mathbb{C}^{N}$ are vectors comprised of noise voltages observed at the antenna array and radio channel transfer functions, respectively, $1 \leq n \leq N$ is an index of an MS antenna. The $n$-th entry of $\boldsymbol{h}$ is given by

$$
h_{n}=\sum_{l=1}^{N_{\mathrm{p}}} \boldsymbol{E}_{\mathrm{m}, n}^{\mathrm{H}}\left(\phi_{l}, \theta_{l}\right) \boldsymbol{\alpha}_{l} \boldsymbol{E}_{\mathrm{b}} e^{\mathrm{j} \xi_{l}},
$$

where $\boldsymbol{E}_{\mathrm{m}, n}$ is the polarimetric complex radiation pattern of the $n$-th antenna, ${ }^{\mathrm{H}}$ is Hermitian transpose. See Appendix of [6] for their definitions; $\boldsymbol{E}_{\mathrm{b}}=\left[\begin{array}{ll}1 & 1\end{array}\right]^{\mathrm{T}} / \sqrt{2}$ or $\left[\begin{array}{ll}1 & 0\end{array}\right]^{\mathrm{T}}$ represents an ideal dual and single polarized omni-directional antenna at BS with consistent total transmit power, $\xi$ is a uniformly distributed random phase over $[02 \pi)$, which is set to 0 for a line-of-sight path; ${ }^{\mathrm{T}}$ denotes transpose. Adding

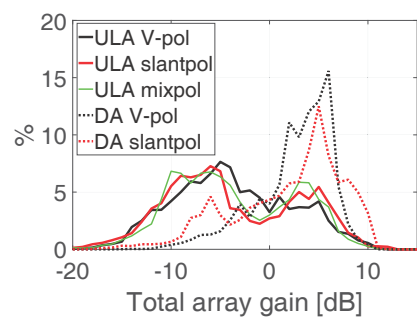

(a)

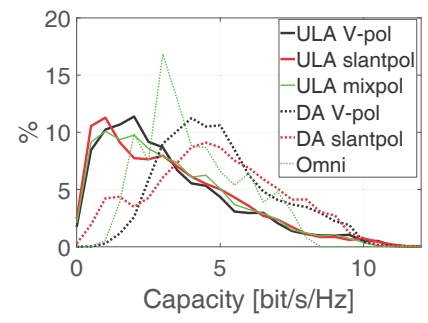

(b)
Fig. 6. Histogram of (a) total array gain and (b) narrowband channel capacity under the presence of human torso and finger shadowing. A dual-polarized omni-directional base station antenna array is considered.

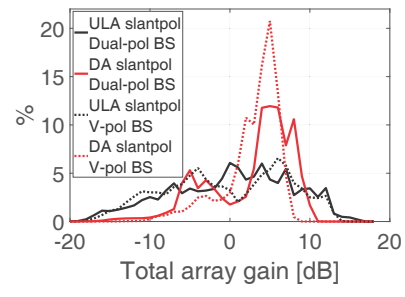

(a)

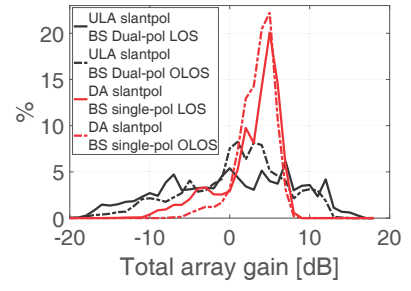

(b)
Fig. 7. Histogram of total array gain and under the presence of human torso, without finger effects. (a) Comparison of gains when single or dual polarized omni-directional antenna is installed at the base station. (b) Comparison of LOS and OLOS channels.

the random phase $\xi$ leads to small-scale realizations of $\boldsymbol{h}$. We consider maximum ratio combining (MRC) assuming that a moving speed of MS is modest so that instantaneous channel information is available at MS, and that the link is noise-limited and hence MS aims at maximizing a signalto-noise ratio (SNR). The combining weights are given by $\boldsymbol{w}=\boldsymbol{h}^{H} /\|\boldsymbol{h}\|$, leading to the total array gain as,

$$
G_{\mathrm{a}}=10 \log _{10}\left(E_{\boldsymbol{h}}\left[|\boldsymbol{h} \boldsymbol{w}|^{2}\right] / P_{\mathrm{o}}\right) \mathrm{dB},
$$

where $E_{\boldsymbol{h}}[\cdot]$ is the Ensemble averaging over small-scale realizations of $\boldsymbol{h}$ and $P_{\mathrm{o}}$ is an omni-directional link gain as

$$
P_{\mathrm{o}}=\sum_{l=1}^{N_{\mathrm{p}}}\left|\boldsymbol{\alpha}_{l}\right|^{2}
$$

for an MS location. The per-path polarimetric complex amplitude $\boldsymbol{\alpha}$ includes the free space and shadowing loss due to small blocking objects and a human torso, as defined in (1). The total array gain includes averaged gains of all antenna elements in the array, as well as precoding and combining gains. The gain is distinct from the conventional array gain in that its value is defined uniquely; the conventional gain values depend on the reference antenna element in an array. The total array gain allows fair comparison of phased antenna arays consisting of different element types and configurations.

Finally, we define the noise level at an MS so that a link with the largest omni-directional pathloss $P_{\mathrm{m}}=\min \left(P_{\mathrm{o}}\right)$ in our considered channel leads to $0 \mathrm{~dB}$ SNR. Assuming that a channel is flat-fading thanks to the beamforming at MS, a 
narrowband capacity of the beamformed channel is given by

$$
C=\log _{2}\left(1+E_{\boldsymbol{h}}\left[|\boldsymbol{h} \boldsymbol{w}|^{2}\right] / P_{\mathrm{m}}\right) .
$$

The capacity is defined by a mean SNR over small-scale fading realizations of channels for a specific orientation of MS.

\section{B. Results and Discussions}

Figure 6(a) shows a histogram of total array gain (9) across different MS locations as well as orientations of the phone chassis at each location. The figure shows that gain can take negative values due to body torso and finger shadowing. The shadowing effect is more pronounced in ULA than DA, where the histogram of ULA peaks around $-5 \mathrm{~dB}$ in addition to that at $5 \mathrm{~dB}$. The bimodal distributions are partly due to the multipath channel characteristics of the considered small-cell scenario. The histogram tends to peak at the higher total array gain for ULA with slanted polarizations than for ULA with vertical polarizations and mixture of vertically and horizontal polarizations. The ULA never achieves the maximal total array gain of $G_{\mathrm{b}}+10 \log _{10} 8=17.2 \mathrm{~dB}$ in our study as a human torso always block the LOS between the antenna array and BS. The histogram of gains from DA shows clear maxima at positive gains, indicating its robustness compared to ULA. DA with slanted polarization shows similar histogram to that with vertical polarizations.

Figure 6(b) depicts a histogram of the narrowband channel capacity (11) estimated from the total array gain and $P_{\mathrm{m}}=-105 \mathrm{~dB}$ in the considered small-cell scenario. The capacity scales logarithmically to the total array gain. The ratio of mean capacity for DA and ULA, $\bar{C}_{\mathrm{DA}} / \bar{C}_{\mathrm{ULA}}$ is about 1.6, showing implications of DA's capability to capture more energy than ULA on link performance. Calculation of total array gain and capacity does not consider possible losses of feeding lines between antenna ports and a receiver as losses depend on implementation. If feeding losses are considered in DA only, the tolerable loss is up to about $5 \mathrm{~dB}$ so that its mean capacity equals to that of ULA without feeding losses.

Figure 7(a) compares the total array gain when omnidirectional antenna BS is single or dual polarized. The former corresponds to the vertical polarization and the latter to horizontal polarization in addition. The total transmit power from the two cases is identical. Finger shadowing effects are not included when deriving the mentioned results in Fig. 7(a), but the same insights are obtained when finger shadowing effects are considered. The gain differences due to polarization states at the base station is insignificant for our small-cell site and studied MS antenna arrays. Even though BS and MS antenna arrays are single polarized, the random orientation of MS ensures some received power after signal combining. Finally, Fig. 7(b) shows gains of the same antenna array in LOS and OLOS conditions. The curves indicate that gain distributions depend on array configurations, i.e., ULA or DA, and not on link conditions of our study.

\section{CONCLUDING REMARKS}

The present paper quantified gains of MS antenna arrays at $60 \mathrm{GHz}$ equipped with ideal analog beamforming and a single transceiver chain. The total array gain is studied to circumvent the ambiguity of conventional array gains depending on the choice of a reference antenna element in an array. Our analysis revealed that the gain is higher for 8-element DA than ULA with the same number of antennas, leading to $60 \%$ increase of the mean channel capacity under realistic noise level setting. Under the presence of human torso and finger operating MS, ULA of the present study never achieves the maximum total array gain. Up to about $5 \mathrm{~dB}$ feeding losses are acceptable in DA so that its mean capacity is comparable to that of ULA without feeding losses. The gains depend mainly on the geometrical configurations of the array, and not on their polarization states and existence of LOS in the link, indicating optimal placement of antenna elements is most important aspect of further research for a phased antenna array with a single transceiver chain.

\section{ACKNOWLEDGEMENT}

The authors would like to acknowledge the financial support from the Academy of Finland research project "Massive MIMO: Advanced Antennas, Systems and Signal Processing at mm-Waves (M3MIMO)" (decision \#288670).

\section{REFERENCES}

[1] 3GPP, TR 38.901 (V14.3.0), "Study on channel model for frequency spectrum from 0.5 to $100 \mathrm{GHz}$,' http://www.3gpp.org/dynareport/38901.htm, Dec. 2017

[2] J. Hejselbaek, J. Ø. Nielsen, W. Fan, and G. Pedersen, "Measured 21.5 $\mathrm{GHz}$ indoor channels with user-held handset antenna array," IEEE Trans. Ant. Prop., vol. 65, no. 12, pp. 6574-6583, Dec. 2017.

[3] I. Syrytsin, S. Zhang, G. F. Pedersen, K. Zhao, T. Bolin, and Z. Ying, "Statistical investigation of the user effects on mobile terminal antennas for 5G applications," IEEE Trans. Ant. Prop., vol. 65, no. 12, pp. 65966605, Dec. 2017

[4] K. Zhao, J. Helander, D. Sjberg, S. He, T. Bolin, and Z. Ying, "User body effect on phased array in user equipment for the $5 \mathrm{G}$ mmWave communication system," IEEE Ant. Wireless Prop. Lett., vol. 16, pp. 864-867, 2017.

[5] K. Zhao, C. Gustafson, Q. Liao, S. Zhang, T. Bolin, Z. Ying, and S. He, "Channel characteristics and user body effects in an outdoor urban scenario at 15 and $28 \mathrm{GHz}$," IEEE Trans. Ant. Prop., vol. 65 , no. 12, pp. 6534-6548, Dec. 2017.

[6] K. Haneda, M. Heino, and J. Järveläinen, "Total array gains of millimeter-wave mobile phone antennas under practical conditions," in Proc. IEEE 87th Vehicular Technology Conference (VTC Spring 2018), June 2018, pp. 1-6, available at arXiv e-prints, http://arxiv.org/abs/1802.08591.

[7] R. G. Vaughan and J. B. Andersen, "Antenna diversity in mobile communications," IEEE Trans. Veh. Tech., vol. 36, no. 4, pp. 149-172, Nov 1987.

[8] T. Taga, "Analysis for mean effective gain of mobile antennas in land mobile radio environments," IEEE Trans. Veh. Tech., vol. 39, no. 2, pp. 117-131, May 1990.

[9] J. Hansen, Spherical near-field antenna measurement. IEE Electromagnetic waves series 26, Peter Peregrinus, London, UK, 1998.

[10] M. Heino, C. Icheln, and K. Haneda, "Finger effect on $60 \mathrm{GHz}$ user device antennas," in 10th European Conf. Ant. Prop. (EuCAP 2016), April 2016, pp. 1-5.

[11] J. Järveläinen, K. Haneda, and A. Karttunen, "Indoor propagation channel simulations at $60 \mathrm{GHz}$ using point cloud data," IEEE Trans. Ant. Prop., vol. 64, no. 8, pp. 4457-4467, Aug. 2016.

[12] J. Vehmas, J. Järveläinen, S. Nguyen, R. Naderpour, and K. Haneda "Millimeter-wave channel characterization at helsinki airport in 15, 28 , and $60 \mathrm{GHz}$ bands," in 84th Veh. Tech. Conf. (VTCFall-2016), Montreal, Canada, Sep. 2016, pp. 1-5.

[13] A. Karttunen, C. Gustafson, A. F. Molisch, J. Järveläinen, and K. Haneda, "Censored multipath component cross-polarization ratio modeling," IEEE Wireless Commun. Lett., vol. 6, no. 1, pp. 82-85, Feb. 2017. 